\title{
Multilayer coating facility for the HEFT hard $x$-ray telescope
}

Carsten P. Jensen, Finn Erland Christensen, Hubert C.M. Chen, Erik B. W. Smitt, Eric Ziegler

Carsten P. Jensen, Finn Erland Christensen, Hubert C.M. Chen, Erik B. W. Smitt, Eric Ziegler, "Multilayer coating facility for the HEFT hard $x$-ray telescope," Proc. SPIE 4496, X-Ray Optics for Astronomy: Telescopes, Multilayers, Spectrometers, and Missions, (30 January 2002); doi: $10.1117 / 12.454368$

Event: International Symposium on Optical Science and Technology, 2001, San Diego, CA, United States 


\title{
Multilayer coating facility for the HEFT hard X-ray telescope
}

\author{
Carsten P. Jensen ${ }^{\mathrm{a}}$, Finn E. Christensen ${ }^{\mathrm{a}}$, Hubert Chen ${ }^{\mathrm{b}}$, Erik B. W.Smitt ${ }^{\mathrm{a}}$, Eric Ziegler ${ }^{\mathrm{c}}$ \\ ${ }^{\mathrm{a}}$ Danish Space Research Institute (Denmark); ${ }^{\mathrm{b}}$ California Institute of Technology (USA), \\ ${ }^{c}$ European Synchrotron Radiation Facility (France)
}

\begin{abstract}
A planar magnetron sputtering facility has been established at the Danish Space Research Institute (DSRI) for the production coating of depth graded multilayers on the thermally slumped glass segments which form the basis for the hard $\mathrm{X}$-ray telescope on the HEFT balloon project. The facility is capable of coating 20-45 mirrors segments in each run. The coatings are optimized W/Si coatings. The paper describes the facility, the results of the calibration and presents data for the X-ray testing of flight mirrors.
\end{abstract}

Keywords: Multilayer, Hard X-ray telescope

\section{INTRODUCTION}

The High Energy Focusing Telescope (HEFT) is a balloon borne mission, which employs focusing optics in the hard X-ray band $(20 \mathrm{keV}-100 \mathrm{keV})$ for sensitive observations of astrophysical sources. The primary scientific objectives include imaging and spectroscopy of ${ }^{44} \mathrm{Ti}$ emission in young supernova remnants, and sensitive hard X-ray observations of obscured Active Galactic Nuclei ${ }^{1}$. The focusing optic is based on depth graded multilayer coatings ${ }^{2,3}$ deposited on thermally slumped thin glass mirror segments ${ }^{4,5}$. The HEFT telescope is a conical approximation to a Wolter-I geometry containing 72 mirror shells per telescope module. Each mirror shell is divided into 5 mirror segments. Thus each telescope module is made up of 720 mirror segments. The mirror segments are $200 \mathrm{~mm}$ in the direction of the optical axis and varying in radius from 40 $\mathrm{mm}$ to $120 \mathrm{~mm}$. A single reflection prototype has demonstrated subarcminute-imaging ${ }^{6}$. Once fully instrumented the HEFT balloon payload will carry several telescope modules. This requires that thousands of mirror segments must be coated in a relatively short period of time. To meet this requirement we have at DSRI established a production coating facility. The facility is based on a planar magnetron sputtering arrangement. The next section describes the facility and the following section gives an example of both soft and hard X-ray reflectance data from a coated flight mirror.

\section{COATING FACILITY}

The facility is a $0.8 \mathrm{~m}^{3}$ vacuum-chamber (bell-jar) with two planar DC magnetron sources. The magnetron sources are placed in the centre of the chamber facing outwards. A ring with a diameter of $1 \mathrm{~m}$ is carrying 10 sample mounting plates which each can contain up to 5 mirror segments depending on the radius of the mirror. The magnetrons are $500 \mathrm{~mm}$ long and $38 \mathrm{~mm}$ wide but only the central $300 \mathrm{~mm}$ of the magnetron can be used due to effects from the ends of the magnets in the magnetron. The magnetrons can rotate $\pm 160^{\circ}$ into a screen (to closed position) from its open position, see Fig. 1a, and the target-sample distance can be varied from $60 \mathrm{~mm}$ to $200 \mathrm{~mm}$. The thickness of the coating will be controlled by the rotation speed of the magnetrons or the ring. The coatings are done with an Ar pressure of $0.53 \mathrm{~Pa}$ (4.0 mTorr) and in most cases with $750 \mathrm{~W}$ applied to both magnetrons. The background pressure are better than $7 * 10^{-5} \mathrm{~Pa}\left(5^{*} 10^{-7}\right.$ Torr $)$. A computer program has been developed to automate the coating.

The system has three different coating modes and a calibration mode. The modes will be described below. For all modes the coating starts with both magnetrons in closed position.

For the first mode the mirror segments will be placed so their optical axis is vertical and the sample-target distance will be adjusted to fit the radius of the mirror segments. A mounting plate will be placed in front of the magnetron and the magnetron will sweep from one closed position to the other. This coating mode corresponds to a line source placed near the 

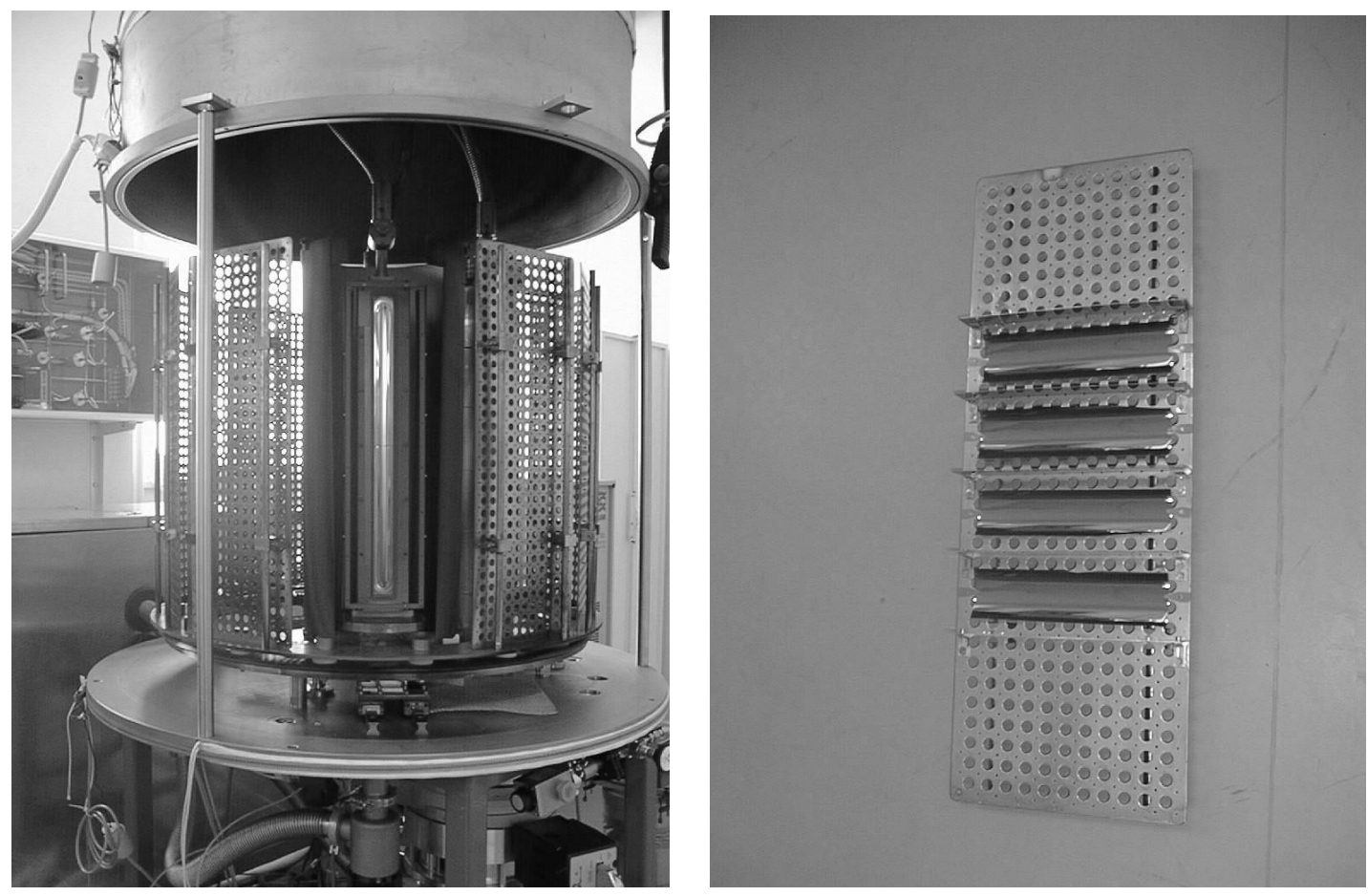

Fig. 1a: The vacuum chamber. One of the 10 mounting plates is removed to expose one of the magnetrons in the open position. b: A mounting plate with four coated segmented mirrors mounted, each with a radius of $40 \mathrm{~mm}$. The mounting plate is $660 \mathrm{~mm}$ tall and $240 \mathrm{~mm}$ wide.

centre of the cylindrically segmented mirror. The sweeping speed will determine the coating thickness. Then the ring is moved to place the next mounting plate in front of the magnetron and the magnetron is sweeped again. After one full rotation of the ring the other material is coated in the same way. This coating mode gives potentially a very high uniformity on the curved segmented mirrors but is time consuming and only one segmented mirror can be mounted on each mounting plate.

For the second mode only 9 of the sample mounting plates are used but the segmented mirrors are mounted with the optical axis horizontal so up to five segmented mirrors can be mounted on each, see Fig. 1b. The empty plate is placed in front of the magnetron, the magnetron is opened, the ring is turned one rotation and the magnetron is closed. The ring is rotated a half rotation so the empty mounting plate is in front of the other magnetron and the next layer is coated. This mode is faster than the previous mode and with some collimation nearly the same homogeneity on the curved substrate can be reached. When using this mode and the previous mode both magnetrons are running at their maximum power compatible with good quality coatings.

For the third coating mode an empty mounting plate is placed in front of both magnetrons. Both magnetrons are opened and when the big ring has made one full rotation a complete bilayer has been coated. The magnetron with the lowest coating rate is running on full power and the thickness is controlled by the rotating speed of the big ring. The power applied to the other magnetron is adjusted so the desirable ratio between the thicknesses of the two materials is obtained. This coating mode is the fastest and the coating time for one run of HEFT mirrors will be between 5 hours and 11 hours.

The calibration mode is designed to make 5 different constant d-spacing coatings in one run. Calibration samples are placed on every second of the mounting plates. An empty mounting plate is placed in front of one of the magnetron and this is opened and 5 different speeds are used to rotate past the 5 calibration samples. In this way 5 different constant d-spacing samples are produced. From X-ray measurements the thickness of the constant d-spacing can be calculated and a calibration file can quickly be generated, see Fig 2 . 


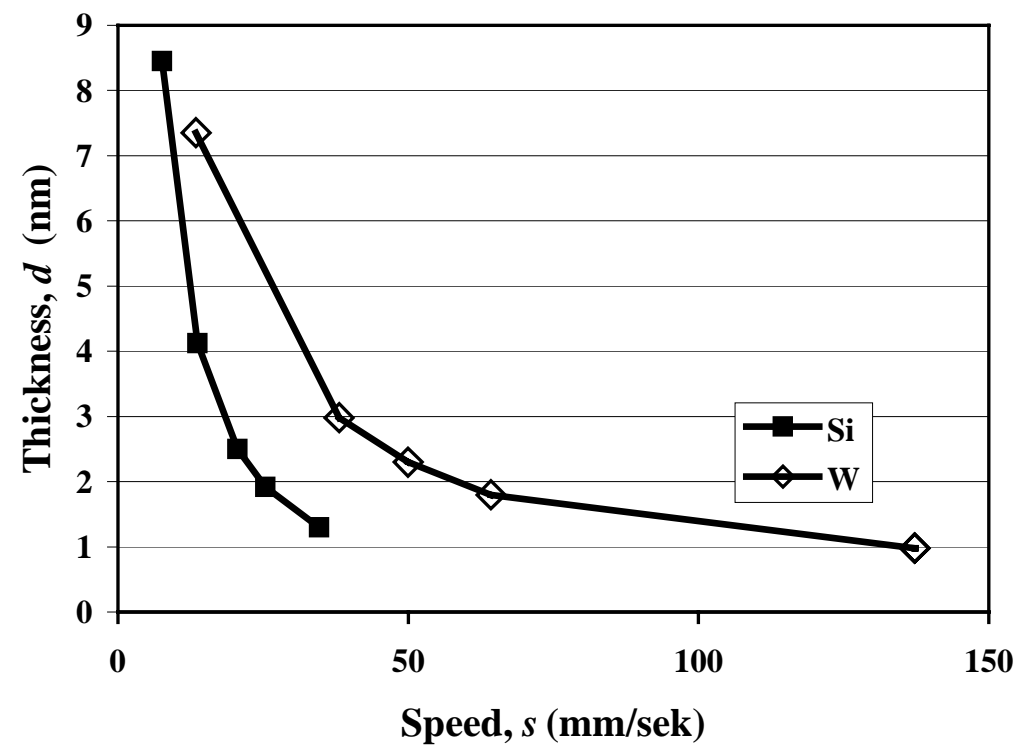

Fig. 2: Coating thickness as function of speed of the ring for both $\mathrm{W}$ and Si running at $750 \mathrm{~W}$ and a target sample distance of $110 \mathrm{~mm}$. Straight lines have been drawn between the points to guide the eye.

\section{X-RAY DATA}

To demonstrate the X-ray reflectance performance from coated flight mirrors we obtained data at $8 \mathrm{keV}$ using a rotating anode facility at DSRI as well as $17 \mathrm{keV}, 40 \mathrm{keV}$ and $60 \mathrm{keV}$ data from the optics beamline (BM5) at the European Synchrotron Radiation Facility. The mirror segment was coated with one of the optimized W/Si multilayer coatings for the HEFT telescopes ${ }^{7}$. The specific coating is the one that will be used for the smallest radius mirror segments. It is a power law grading of the periods ${ }^{8}$. Figure 3 , shows all the reflectance data versus $q$,

$$
q=4 \pi \frac{\sin \Theta}{\lambda}
$$

Each data set has been shifted an order of magnitude for clarity. The full lines represent a fit to the data using the same structural parameters for the multilayer coating. These are a minimum period of $3.50 \mathrm{~nm}$ and a maximum period of 29.66 $\mathrm{nm}$, a ratio between the heavy element thickness to the period of 0.4 , a power law index of 0.236 and an interfacial roughness of $0.40 \mathrm{~nm}$. The roughness of that glass segment was measured to be $0.42 \mathrm{~nm}$ before the coating. A reasonable fit is obtained for all energies.

The azimuthal uniformity on the mirror segments has been studied by X-ray measurements at $8 \mathrm{keV}$ from constant period multilayers. We use constant d-spacing coatings with 10 bilayers because the X-ray data from these coatings are very sensitive to a shift of the d-spacing, so even small variations in the thickness over the azimuth on the segmented mirror will be easily detectable. The center of the segmented mirrors is defined as $\theta=0^{\circ}$ and measurements has been made at $\pm 10^{\circ}$, $\pm 20^{\circ}$ and $\pm 30^{\circ}$. There is as expected good agreement for points that is symmetrical around $\theta=0^{\circ}$. With no collimation the thickness variations were larger than $10 \%$ between $0^{\circ}$ and $30^{\circ}$. Figure 4 shows the thickness variation along the azimuth for three coatings with nominal thickness of $2.2 \mathrm{~nm}, 4.2 \mathrm{~nm}$ and $7.4 \mathrm{~nm}$ for coatings with much improved collimation. The thickness variation along the azimuth is less than $2.5 \%$ for $95 \%$ of the data points. This is well within the specifications for HEFT. By still improving the collimation we expect to make the thickness variation even smaller. There is no variation in the roughness along the azimuth for any of the coatings. 


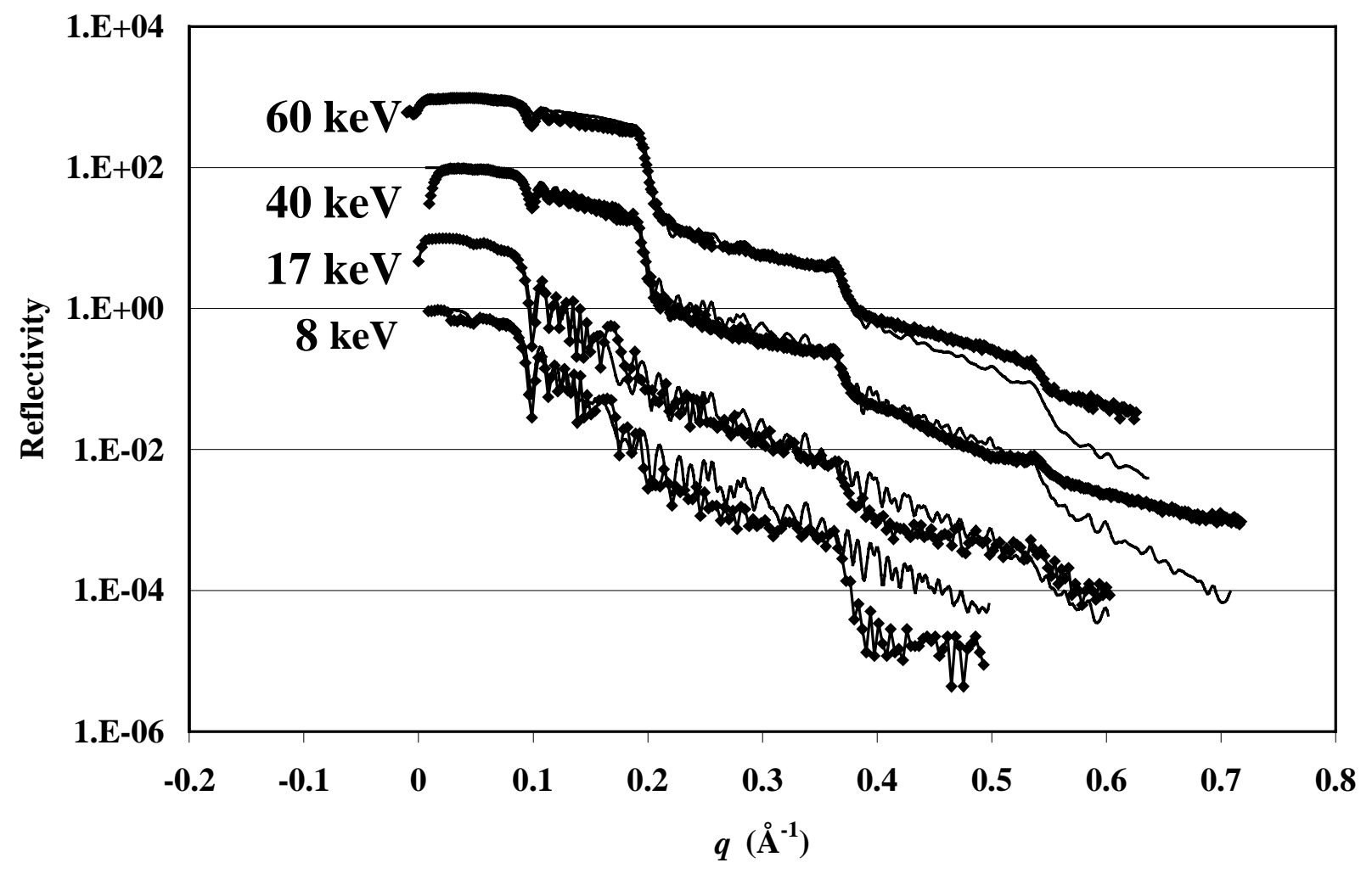

Fig. 3: Reflectivity versus $q$ for $8 \mathrm{keV}, 17 \mathrm{keV}, 40 \mathrm{keV}$ and $60 \mathrm{keV}$ measurements on the same segmented mirror. The thin lines are fits with the same model, only varying the energy. The curves are shifted an order of magnitude for clarity.

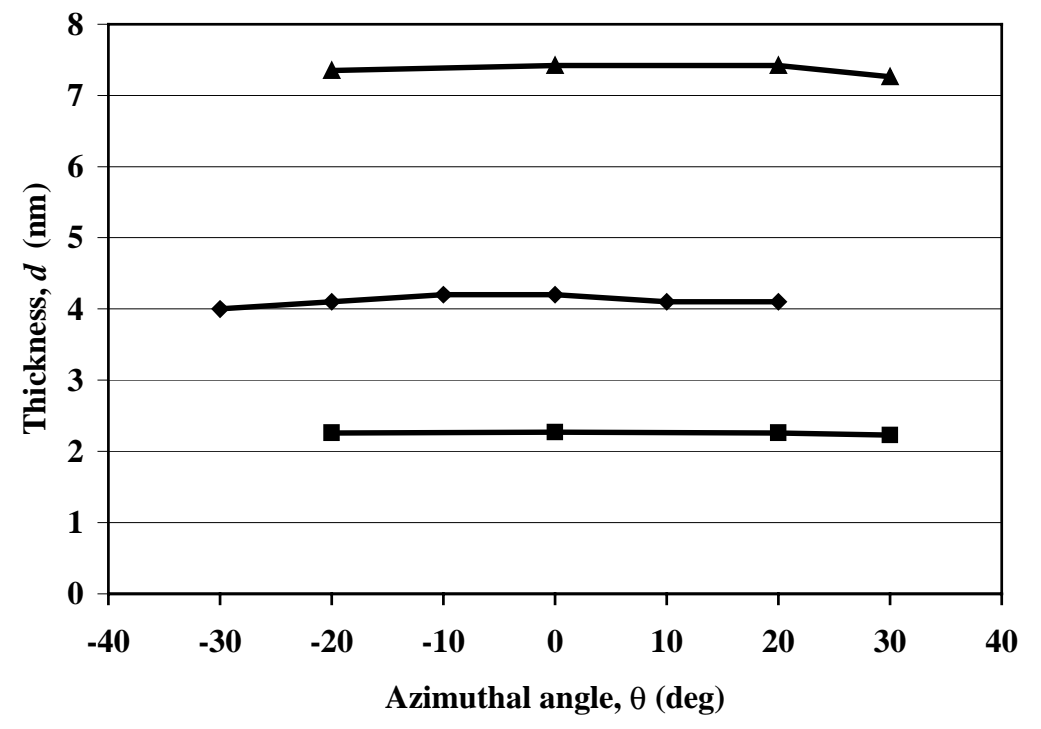

Fig. 4: Bilayer thickness measured from three constant d-spacing coatings on segmented mirrors as function of the azimuth angle. Straight lines have been drawn between the points to guide the eye. 


\section{CONCLUSION}

A multilayer production coating facility capable of coating segmented optics for hard X-ray telescopes such as the HEFT telescopes and the segmented version of the Hard X-ray Telescopes on the Constellation-X has been described. It is currently being used for the W/Si coatings optimized for the HEFT hard X-ray balloon mission. The facility allows for 2045 segments to be coated in one run depending on the radius of the pieces. At this rate all mirror segments for one HEFT telescope module or one HXT/Con-X module can be coated in 1-2 months.

\section{REFERENCES}

1. F. A. Harrison et al, "Development of the High-Energy Focusing Telescope (HEFT) balloon experiment," SPIE Vol. 4012, pp. 693-699, 2000.

2. F. E. Christensen et al, "A graded d-spacing multilayer telescope for high energy X-ray Astronomy," SPIE Vol. 1546, pp. 160-167, 1991.

3. D. L. Windt et al, "Growth, structure and performance of depth-grated W/Si multilayers for hard X-ray optics," J. Applied Physics, Vol. 88, pp. 460-470, 2000.

4. C. J. Hailey et al, "Investigation of substrates and mounting techniques for the High Energy Focusing Telescope (HEFT)," SPIE Vol. 3114, pp. 535-543, 1997.

5. W. W. Craig et al, "Hard X-ray Optics for the HEFT Balloon Borne Payload: Prototype Design \& Status," SPIE Vol. 3445, pp. 112-120, 1998.

6. W. W. Craig et al, "Development of Thermally Formed Glass Optics for Astronomical Hard X-ray Telescopes," Optics Express, Vol. 7, pp. 178-185, 2000.

7. P. H. Mao et al, "Optimization of graded multilayer designs for astronomical x-ray telescopes," Applied Optics, Vol. 38, pp. 4766-4775, 1999.

8. K. D. Joensen et al, "Design of grazing-incidence multilayer supermirrors for hard X-ray reflectors," Applied Optics, Vol. 34, pp. 7935-7944, 1995. 